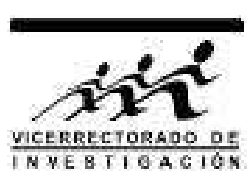

\title{
Comparación de costos de producción conjunta de electricidad y calor usando gas natural y glicerol
}

\author{
C. A. Quispe Gonzáles ${ }^{* 1,2}$, W. E. Alvarado Torres ${ }^{1}$, M. A. Ormeño Valeriano ${ }^{1}$ y R. E. Vargas Roncal ${ }^{1}$ \\ ${ }^{1}$ Facultad de Ciencias Físicas, Universidad Nacional Mayor de San Marcos, Lima, Perú \\ ${ }^{2}$ Faculdade de Engenharia de Guaratinguetá, Universidade Estadual Paulista, 12516-41, Guaratinguetá, \\ SP, Brasil
}

Recibido 26 setiembre 2012 - Aceptado 15 diciembre 2012

\begin{abstract}
En este trabajo se presenta un análisis termodinámico de un sistema de cogeneración simplificado para la producción combinada de electricidad y calor de proceso. Dadas las condiciones de demandas energéticas internas (electricidad y vapor de proceso) se propone un esquema termodinámico a base de una turbina de vapor trabajando en condiciones de paridad térmica. Se describe la metodología de los cálculos termodinámicos y termoeconómicos del sistema. Son comparados los índices termoeconómicos utilizando dos tipos de combustible: gas natural y glicerol bruto (metilado) y a través de los resultados obtenidos, es posible comparar los precios de producción conjunta de electricidad y calor para los dos tipos de combustibles propuestos.
\end{abstract}

Palabras claves: Análisis termoeconómico, cogeneración, costos de producción electricidad-calor, glicerol.

\section{Joint production cost comparison of electricity and heat using natural gas and glycerol}

This work shows a thermodynamical analysis of a simplified cogeneration system for combined production of electricity and proccess heat. Due to increase of energetic demands, we propose a themodynamics schema based on a steam turbin working in conditions of thermic parity. We describe the thermal and thermal-economics calculations methodology of the system. We compare the thermaleconomics indexes using two types of combustible: natural gas and glycerol (methyled), and through the results obtained, is possible to compare the joint production prices of electricity and heat for the two combustibles proposed.

Keywords: Thermoeconomics analysis, cogeneration, production costs of electricity-heat, glycerol.

La crisis energética por la que actualmente atraviesa el mundo, está relacionada fundamentalmente a los precios fluctuantes del petróleo, a las inestabilidades políticas en los países productores y a las fuertes presiones ambientales a fin de disminuir la concentración de gases de efecto invernadero y la contaminación ambiental. Esto crea un escenario en donde se intensifica la búsqueda de nuevas fuentes energéticas y la optimización de los actuales sistemas energéticos. La creciente demanda de energía, que acompaña el desarrollo económico de los países conlleva a proponer dos tipos de medidas para satisfacer esta demanda: el aumento de la potencia instalada y la optimización de los sistemas de generación. En este contexto, fueron efectuadas muchas

*cquispeg@yahoo.com investigaciones buscando perfeccionar y mejorar la eficiencia de los sistemas energéticos que presenten bajos niveles de inversión, aprovechando al máximo la energía de los combustibles [1-12].

Una de estas tecnologías es la cogeneración, la cual tiene beneficios importantes, sobre todo, en las aplicaciones donde existe una amplia y continua demanda de calor. Su desarrollo ha permitido tener diferentes tecnologías comprobadas para la producción combinada de electricidad y calor, utilizando diversos tipos de combustibles.

Entre los combustibles, el precio del gas natural sufrió un incremento significativo durante la crisis del petróleo en el 2008, pero según la Agencia Internacional 
de Energía (International Energy Agency, IEA) predice que los precios internacionales del gas natural comenzará a disminuir en el futuro, como consecuencia de la crisis económica mundial, de los planes de ahorro de energía y del desarrollo de la producción de electricidad a base de energía renovable. Existe la posibilidad que para el 2015 exista un exceso de capacidad instalada de gasoductos y terminales de gas natural licuado (GNL).

Por otro lado, el uso de los biocombustibles introdujo una serie de beneficios, incluyendo la sustentabilidad, la reducción de gases de efecto invernadero, el desarrollo regional, social y agrícola [13, pero también introdujo un subproducto no tan deseado como el glicerol. En general, cerca de $10 \mathrm{~kg}$ de glicerol son obtenidos por cada $100 \mathrm{~kg}$ de biodiesel [14]. En los últimos años la producción mundial de biodiesel ha crecido exponencialmente [15] y recientemente ha aumentado de manera significativa [16]. A partir del 2005 los precios del glicerol bruto comenzaron a caer debido a la gran producción de biodiesel que incorporó grandes cantidades de glicerol a los mercados 17.

La viabilidad de los sistemas de cogeneración está asociada a preguntas sobre los índices energéticos y los costos de producción de electricidad y vapor. Son dos los regímenes de trabajo más comunes en la cogeneración: la paridad eléctrica, que es más fácil de satisfacer, ya que solo basta con elegir el equipo de generación de los catálogos propuestos por los fabricantes y la paridad térmica, la cual requiere de un esquema termodinámico óptimo y del cálculo de los índices energéticos y termoeconómicos que justifiquen la viabilidad de estos sistemas. La determinación de los costos de la producción de electricidad y la producción de vapor de proceso están relacionados directamente al tipo de combustible usado en la instalación, lo que es evaluado en este trabajo.

\section{Esquema termodinámico y demandas ener- géticas}

Se presenta el caso de satisfacer las demandas de electricidad y de calor de una planta de producción de papel. El esquema termodinámico del sistema energético propuesto se muestra en la Fig. 1 y las demandas energéticas son: (1) Consumo de vapor de proceso de $100 \mathrm{Tn} / \mathrm{h}$, a una presión de $0.24 \mathrm{MPa}$, (2) Demanda eléctrica interna de $6000 \mathrm{~kW}$, y (3) Combustibles a considerar: Gas natural (GN) con poder calorífico interior, PCI, de $48 \mathrm{MJ} / \mathrm{kg}$, y el glicerol con PCI de 15 $\mathrm{MJ} / \mathrm{kg}$.

En este esquema, la caldera GV produce vapor recalentado que es dirigido a una turbina de vapor TV, en donde se expande transformando la energía térmica en energía eléctrica con ayuda de un generador eléctrico G, unido a la turbina. Una parte del vapor que atraviesa la turbina, es extraído a parámetros determi- nados y utilizado como vapor de proceso, para satisfacer la demanda de calor en diversos procesos industriales, expandiéndose hasta condiciones de líquido saturado. Luego es dirigido a un regenerador de mezcla. A su vez, la otra parte de vapor, pasa por toda la turbina y una vez agotado, es dirigido hacia el condensador $\mathrm{C}$ donde se condensa, pasando luego al regenerador de mezcla. La bomba B toma el fluido del regenerador y lo envía hacia la caldera.

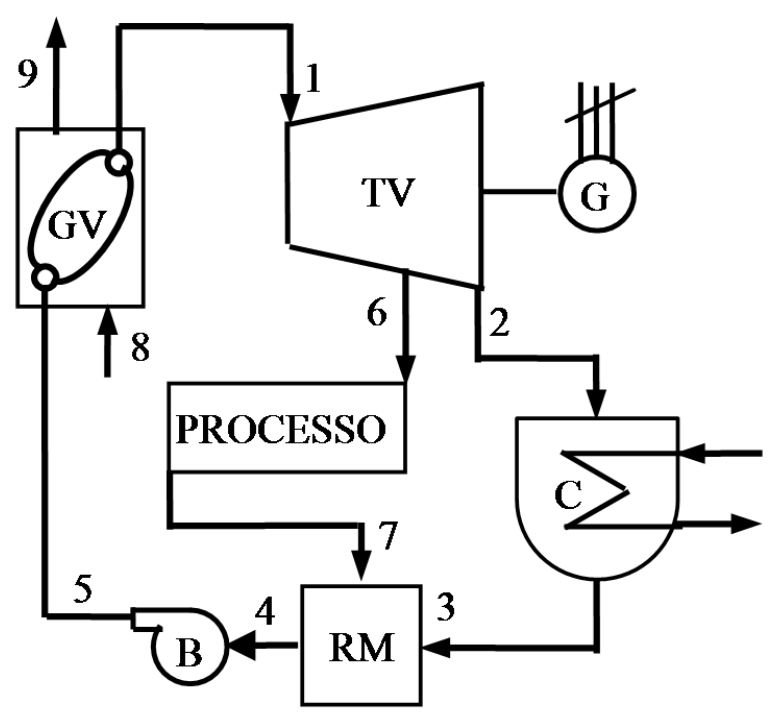

Figura 1: Esquema termodinámico del sistema de cogeneración.

\section{Metodología del cálculo térmico}

De acuerdo al esquema termodinámico propuesto y a los datos de las demandas energéticas, el sistema trabaja bajo un ciclo Rankine. Los parámetros termodinámicos isoentrópicos en cada uno de los puntos del esquema, fueron determinados con ayuda de calculadores de vapor [18] y se muestran en la Tabla 1.

Para determinar el caudal de vapor se tomó una reserva de $20 \%$ en función a las calderas disponibles en el mercado y considerando el incremento futuro de las demandas de vapor. La caldera propuesta tiene una producción de vapor de $120 \mathrm{Tn} / \mathrm{h}$, la presión de vapor es $4.5 \mathrm{MPa}$ a una temperatura de $410^{\mathrm{O}} \mathrm{C}$. El condensador trabaja a una presión constante de $12 \mathrm{kPa}$. La presión desarrollada por la bomba de alimentación fue incrementada en $20 \%$ para asegurar la presión del vapor, superar las pérdidas hidráulicas en la caldera, en las tubuladuras de conducción y en la válvula de entrada a la turbina a vapor. La determinación de los parámetros de los gases de escape de la caldera, de los caudales del combustible y valores de gasto de combustible y de los gases de combustión son determinados más adelante. 
Rev. Inv. Fis. 15, 121501651 (2012)

Tabla 1: Parámetros termodinámicos en los puntos característicos del ciclo de vapor.

\begin{tabular}{ccccccccc}
\hline Punto & Fluido & $\dot{\mathrm{m}}(\mathrm{kg} / \mathrm{s})$ & $\mathrm{P}(\mathrm{MPa})$ & $\mathrm{T}\left({ }^{\circ} \mathrm{C}\right)$ & $\mathrm{v}\left(\mathrm{m}^{3} / \mathrm{kg}\right)$ & $\mathrm{h}(\mathrm{kJ} / \mathrm{kg})$ & $\mathrm{s}(\mathrm{kJ} / \mathrm{kg}-\mathrm{K})$ & $\mathrm{ex}(\mathrm{kJ} / \mathrm{kg})$ \\
\hline 1 & vapor & 33.333 & 4.50000 & 410.00 & 0.066 & 3229.56 & 6.7423 & 2710.410 \\
2 & vapor & 5.556 & 0.01200 & 49.42 & 10.1129 & 2157.17 & 6.7423 & 1638.014 \\
3 & agua & 5.556 & 0.01200 & 49.42 & 0.00101 & 206.91 & 0.6962 & 153.300 \\
4 & agua & 33.333 & 0.10133 & 91.58 & 0.001037 & 383.64 & 1.2109 & 290.400 \\
5 & agua & 33.333 & 5.40000 & 91.92 & 0.001034 & 389.13 & 1.2109 & 295.890 \\
6 & vapor & 27.778 & 0.00000 & 126.07 & 0.7026 & 2585.38 & 6.7423 & 2066.230 \\
7 & aguas & 27.778 & 0.10133 & 99.97 & 0.00104 & 418.99 & 1.3067 & 318.370 \\
\hline 8 & GN & 2.497 & 0.40000 & 25.00 & & & & \\
$8 \mathrm{a}$ & glicerol & 5.818 & 0.50000 & 25.00 & & & & \\
\hline 9 & gases GN & 29.975 & 0.10133 & 150.00 & & & & \\
$9 \mathrm{a}$ & gases Glic & 49.725 & 0.101325 & 150.00 & &
\end{tabular}

\section{Índices energéticos de la turbina a vapor}

Para calcular el trabajo específico desarrollado en la turbina a vapor, se adopta una eficiencia isoentrópica de la turbina a vapor $\eta_{\text {Istv }}=0.86$. La fracción de gasto másico correspondiente al vapor del proceso $y_{\mathrm{Vp}} \mathrm{y}$ la fracción dirigida al condensador $y_{\mathrm{Vc}}$, se determinan por medio de las relaciones 19

$$
\begin{gathered}
y_{\mathrm{Vp}}=\frac{\dot{m}_{\mathrm{Vp}}}{\dot{m}_{\mathrm{V}}} \quad \mathrm{y} \\
y_{\mathrm{Vc}}=\frac{\dot{m}_{\mathrm{V}}-\dot{m}_{\mathrm{Vp}}}{\dot{m}_{\mathrm{V}}}=1-y_{\mathrm{Vp}} .
\end{gathered}
$$

El balance energético del regenerador de mezcla, con eficiencia $\eta_{\mathrm{RM}}=0.99$, está dado por

$$
\eta_{\mathrm{RM}}=\frac{h_{4}}{y_{\mathrm{Vp}} h_{2}+y_{\mathrm{Vc}} h_{3}}
$$

y la energía específica disponible del vapor al atravesar la turbina a vapor $h_{\mathrm{V}}$ se determina mediante la expresión

$$
h_{\mathrm{V}}=\left(h_{1}-h_{6}\right)+y_{\mathrm{Vc}}\left(h_{6}-h_{2}\right) .
$$

La potencia desarrollada por el vapor al pasar por la turbina a vapor está dado por

$$
P_{\mathrm{TV}}=\dot{m}_{\mathrm{V}} h_{\mathrm{V}} \eta_{\mathrm{Istv}}
$$

y el trabajo requerido por la bomba de alimentación, bajo una eficiencia isoentrópica de $\eta_{\text {Isb }}=0.82$, se determina mediante

$$
P_{\mathrm{B}}=\frac{\dot{m}_{\mathrm{V}}\left(h_{5}-h_{4}\right)}{\eta_{\mathrm{Isb}}}
$$

Determinación de los gastos másicos de combustibles considerados y gases de combustión

La determinación del gasto másico de los combustibles considerados, necesita tomar en cuenta la eficiencia de la caldera, que considera factores como la eficiencia del proceso de combustión, las pérdidas por contenido de hidrógeno en el combustible, las pérdidas por radiación y por convección, por purga, entre otros. Por lo general, la eficiencia global de una caldera $\eta_{\text {Cal }}$ se encuentra en el rango de 77 a $80 \%$. Asumiendo una eficiencia de $80 \%$ tenemos que

$$
\eta_{\mathrm{Cal}}=\frac{\dot{m}_{\mathrm{V}}\left(h_{1}-h_{5}\right)}{\dot{m}_{\mathrm{Comb}} \mathrm{PCI}_{\mathrm{Comb}}} .
$$

El presente trabajo evalúa el uso de dos combustibles, gas natural y glicerol bruto (metilado); para comparar los costos de producción de electricidad y calor. El gas natural puede aproximarse como una mezcla de $92 \%$ de metano y $8 \%$ de propano, que son sus componentes principales. El glicerol en estado puro $\mathrm{C}_{3} \mathrm{H}_{5}(\mathrm{OH})_{3}$ o glicerinas refinadas con alto contenido de glicerol no son de interés, debido a su alto costo de producción para las diferentes etapas de los procesos de purificación, así como su alta viscosidad que dificulta el proceso de atomización previa a la quema. Es de mayor interés el glicerol bruto (metilado) obtenido como residuo directamente de la producción de biodiesel y su composición depende principalmente de la materia prima utilizada y del proceso de producción del biodiesel. Una composición en peso del glicerol metilado es dada en [20], conteniendo $42.5 \%$ de $\mathrm{C} ; 10.14 \%$ de $\mathrm{H}$; $<0.05 \%$ de $\mathrm{N}$ y $43.32 \%$ de O, $0.078 \%$ de S, $1.03 \%$ de $\mathrm{H}_{2} \mathrm{O}, 3.06 \%$ de cenizas y otros componentes particulados $(\mathrm{Ca}, \mathrm{Na}, \mathrm{K}, \mathrm{Cl}, \mathrm{Mg}, \mathrm{P})$. El PCI para esta muestra fue de $21.8 \mathrm{MJ} / \mathrm{kg}$.

La reacción de combustión estequiométrica del gas natural y glicerol bruto propuestos pueden expresarse 
como

$$
\begin{array}{r}
0.92 \mathrm{CH}_{4}+0.08 \mathrm{C}_{3} \mathrm{H}_{8}+2.24\left(\mathrm{O}_{2}+3.76 \mathrm{~N}_{2}\right) \rightarrow \\
1.16 \mathrm{CO}_{2}+2.16 \mathrm{H}_{2} \mathrm{O}+8.4224 \mathrm{~N}_{2}
\end{array}
$$

$\mathrm{y}$

$$
\begin{gathered}
3.504 \mathrm{C}+5.07 \mathrm{H}_{2}+0.0018 \mathrm{O}_{2}+0.0016 \mathrm{~S}+0.057 \mathrm{H}_{2} \mathrm{O}+ \\
4.6866\left(\mathrm{O}_{2}+3.76 \mathrm{~N}_{2}\right) \rightarrow 3.504 \mathrm{CO}_{2}+5.127 \mathrm{H}_{2} \mathrm{O}+ \\
17.6234 \mathrm{~N}_{2}+0.0016 \mathrm{SO}_{2} \quad, \quad \text { (9) }
\end{gathered}
$$

respectivamente.

La combustión estequiométrica es ideal, en condiciones reales los combustibles siempre son quemados bajo un exceso de aire $\alpha$, para lograr una buena mezcla aire/combustible y un proceso eficiente de combustión. El valor de $\alpha$ requerido por una caldera varía de acuerdo al tipo de combustible y a la tecnología del quemador. Para quemadores de gas natural, $\alpha$ se encuentra en un intervalo de 10 a $15 \%$; mientras que para combustibles líquidos viscosos (caso del glicerol), utilizando atomizadores de presión, $\alpha$ está en un intervalo de 15 a $20 \%$. Así, las reacciones del gas natural con $\alpha$ de $15 \%$ y glicerol metilado con $\alpha$ de $18 \%$ están dadas por

$$
0.92 \mathrm{CH}_{4}+0.08 \mathrm{C}_{3} \mathrm{H}_{8}+1.15 \times 2.24\left(\mathrm{O}_{2}+3.76 \mathrm{~N}_{2}\right)
$$$$
\rightarrow \text { productos }(10)
$$

y

$$
\begin{array}{r}
3.504 \mathrm{C}_{2}+5.07 \mathrm{H}_{2}+0.00018 \mathrm{~N}_{2}+1.354 \mathrm{O}_{2}+0.0016 \mathrm{~S}+ \\
0.057 \mathrm{H}_{2} \mathrm{O}+5.5302\left(\mathrm{O}_{2}+3.76 \mathrm{~N}_{2}\right) \rightarrow \text { productos, }
\end{array}
$$

respectivamente.

Las reacciones (10) y (11) permiten calcular la masa de aire que interviene en cada reacción. La masa de gases puede considerarse como la suma de la masa de aire y la masa del combustible. Así, de la reacción (10) se observa que $1 \mathrm{~kg}$ de gas natural necesitará $11.2 \mathrm{~kg}$ de aire y de la reacción (11), $1 \mathrm{~kg}$ de glicerol metilado se necesitará $7.7 \mathrm{~kg}$ de aire. Los resultados obtenidos, corresponden a los puntos 8 y 9 del esquema termodinámico (Fig. 1 y Tabla 1).

\section{Producción y eficiencias del sistema termo- energético}

La producción de energía eléctrica desarrollada por el sistema, considerando la eficiencia del acoplamiento entre la turbina y el generador, $\eta_{\text {Acop }}=0.98$ y la eficiencia del generador eléctrico $\eta_{\mathrm{GE}}=0.99$ está dado por

$$
E_{\mathrm{Elec}}=\left(P_{\mathrm{TV}}-P_{\mathrm{B}}\right) \eta_{\mathrm{Acop}} \eta_{\mathrm{GE}}
$$

mientras que, la producción térmica de vapor de proceso es determinada mediante la relación

$$
E_{\mathrm{T}}=\dot{m}_{\mathrm{VP}}\left(h_{8}-h_{7}\right)
$$

la energía aportada por el combustible está dada por

$$
E_{\mathrm{Comb}}=\dot{m}_{\mathrm{Comb}} \mathrm{PCI}_{\mathrm{Comb}}
$$

y la eficiencia de producción eléctrica $\eta_{\text {Elec }}$, la eficiencia térmica (vapor de proceso) $\eta_{\mathrm{T}}$ y la eficiencia global (electricidad + vapor de proceso) $\eta_{\text {Glob }}$ están dada por

$$
\begin{aligned}
\eta_{\text {Elec }} & =\frac{E_{\text {Elec }}}{E_{\text {Comb }}}, \\
\eta_{\mathrm{T}} & =\frac{E_{\mathrm{T}}}{E_{\mathrm{Comb}}}, \\
\eta_{\mathrm{Glob}} & =\frac{E_{\mathrm{Elec}}+E_{\mathrm{T}}}{E_{\mathrm{Comb}}}=\eta_{\text {Elec }}+\eta_{\mathrm{T}} .
\end{aligned}
$$

Trabajando el sistema en paridad térmica, asegurando la necesidad de vapor de proceso, los resultados arrojan una producción eléctrica de aproximadamente $19.75 \mathrm{MW}$.

\section{Costos de equipamiento e inversión de la planta}

Uno de los componentes más importantes en el análisis económico del sistema está referido a los costos de los equipos y los subsistemas que se presentan a continuación en la Tabla 2.

Tabla 2: Relaciones para la estimación de costos de los equipos del sistema térmico

\begin{tabular}{llr}
\hline Equipo & Expresión de cálculo & Costo del caso (US\$) \\
\hline Caldera, Q en kW & $C_{\mathrm{C}}=768 \times Q^{0.78}$ & $6^{\prime} 957,224.90$ \\
Turbina a vapor & $C_{\mathrm{TV}}=6000 \times E_{\mathrm{Elec}}^{0.7}$ & $6 ' 103,640.51$ \\
Condensador & $C_{\mathrm{Cond}}=1773 \times \dot{m}_{\mathrm{V}}$ & $35,460.00$ \\
Bomba de alimentación & $C_{\mathrm{B}}=3540 \times P_{\mathrm{B}}^{0.71}$ & $164,569.15$ \\
Regenerador de mezcla & $C_{\mathrm{Reg}}=887 \times \dot{m}_{\mathrm{V}}$ & $106,440.00$ \\
Costo de equipamiento de planta & $C_{\text {Equip }}=C_{\mathrm{TV}}+C_{\mathrm{C}}+C_{\mathrm{B}}+C_{\text {Cond }}+C_{\text {Reg }}$ & $13^{\prime} 367,334.60$ \\
\hline
\end{tabular}


Generalmente, el costo de los equipos mecánicos considera el costo de instalación en la planta, los sistemas eléctricos, sistemas de control, protección contra incendio, aislamiento acústico y otros afines. Para determinar estos costos de inversión se pueden utilizar las ecuaciones desarrolladas en [7] las cuales posteriormente fueron adaptadas y mejoradas por otros investigadores $[10,12,21,23]$.

El costo de inversión de la planta se puede considerar como la suma de los costos de los equipos principales del sistema, incrementado en un $30 \%$ y este incremento corresponde a los costos de terreno, transporte, administración de obras, proyectos de ingeniería y otros [10, siendo expresado como:

$$
I_{\mathrm{Pl}}=1.30 \times C_{\mathrm{Eq}} .
$$

\section{Costos de mantenimiento}

El costo de mantenimiento puede ser expresado como una función de la suma de los costos de mantenimiento de cada uno de los equipos que conforman el sistema térmico (turbina a vapor, condensador, regenerador, bomba de alimentación y otros menores). En los análisis preliminares, el costo de mantenimiento puede estimarse entre el 2 y el $10 \%$ de la inversión total en equipos. El menor valor corresponde a grandes sistemas de potencia, mientras que los mayores valores, están asociados a los pequeños sistemas. Para el presente caso, un valor de $6 \%$ puede ser una estimación inicial razonable

$$
I_{\mathrm{Man}}=0.06 \times C_{\mathrm{Eq}} .
$$

\section{Costos de operación del sistema}

En los casos simples, el costo de operación suele basarse en el costo del personal para la operación de la planta, tales como los ingenieros, los técnicos, los administrativos y el personal de servicios. No necesariamente, todo este personal debe estar permanentemente en planta. El valor promedio de sueldo del personal puede estimarse en $S_{\text {pers }}=30000$ US $\$$ /pers-año, aquí se incluyen el pago mensual, los seguros y los beneficios sociales. Como el sistema energético es de pequeña potencia, se podría estimar una necesidad de personal de 4 pers/turno y la planilla total del personal de operación se estima en

$$
N_{\text {pers }}=\frac{24 \frac{\mathrm{h}}{\text { día }} \times 7 \frac{\text { días }}{\text { sem }} \times 4 \frac{\text { pers }}{\text { turno }}}{40 \frac{\mathrm{h}}{\text { sem-turno }}}=17 \text { pers } .
$$

Así, el costo de operación estará definido por la expresión

$$
C_{\mathrm{Op}}=N_{\mathrm{pers}} \times S_{\mathrm{pers}}
$$

Para valorar la influencia de la inversión se utiliza el concepto de factor de anualidad pay-back, plazo de recuperación o factor de anualidad, la cual viene dada por la siguiente expresión

$$
f=\frac{q^{k}(q-1)}{q^{k}-1} \quad \text { donde } \quad q=1+\frac{r}{100}
$$

donde $k=7$ años es el periodo de amortización o pay $b a c k$, plazo de recuperación de la inversión, $r=12 \%$ es la tasa de interés anual, que depende del país donde se produce la inversión.

\section{Determinación de los costos de producción de electricidad y calor}

El sistema térmico propuesto produce dos productos: energía eléctrica y energía térmica (vapor de proceso), utilizando conjuntamente gran parte de los equipos del sistema. Con el fin de valorar adecuadamente el costo de producción de cada uno de estos productos, se introduce el concepto del factor de producción que representa la fracción de producción de cualquiera de las formas de energía respecto a la producción global de energía. Así, se define el factor de producción de energía eléctrica $F_{\text {Elec }}$ y el factor de producción de energía térmica $F_{\mathrm{T}}$ por las siguientes relaciones,

$$
\begin{aligned}
F_{\text {Elec }} & =\frac{E_{\text {Elec }}}{E_{\text {Elec }}+E_{\mathrm{T}}} \\
F_{\mathrm{T}} & =\frac{E_{\mathrm{T}}}{E_{\text {Elec }}+E_{\mathrm{T}}},
\end{aligned}
$$

es evidente que $F_{\text {Elec }}+F_{\mathrm{T}}=1.0$.

El costo del combustible es un componente variable ya que los precios internacionales varían de acuerdo a las condiciones y a los escenarios de la producción y la demanda mundial. El costo de combustible, en el caso del gas natural GN, ha sufrido una disminución en los últimos años, estando ahora entre 0.0025 a 0.005 $\mathrm{US} \$ / \mathrm{MJ}$, lo que produce de 0.009 a $0.015 \mathrm{US} \$ / \mathrm{kW}-\mathrm{h}$. En el caso del glicerol, que actualmente es un subproducto muy barato en la producción de biodiesel, es de 40 a 60 US $\$ /$ Ton, lo que produce de 0.007 a 0.00104 $\mathrm{US} \$ / \mathrm{kW}-\mathrm{h}$.

El costo de producción de energía eléctrica y el costo de producción de energía térmica (vapor de proceso) pueden ser evaluados a través de métodos simplificados 10,22. De acuerdo a estas recomendaciones, el costo de energía eléctrica y el costo de energía térmica (vapor de proceso) generados por el sistema energéticos son

$$
\begin{aligned}
C_{\text {Elec }} & =\left[I_{\mathrm{pl}} f+C_{\mathrm{man}}+C_{\mathrm{Op}}\right] \frac{F_{\text {Elec }}}{H E_{\mathrm{Elec}}}+\frac{C_{\mathrm{Comb}}}{\eta_{\mathrm{Elec}}} \\
C_{\mathrm{T}} & =\left[I_{\mathrm{pl}} f+C_{\mathrm{man}}+C_{\mathrm{Op}}\right] \frac{F_{\mathrm{T}}}{H E_{\mathrm{T}}}+\frac{C_{\mathrm{Comb}}}{\eta_{\mathrm{T}}} .
\end{aligned}
$$




\section{Análisis de los resultados}

Los resultados de los costos para la energía eléctrica y para la energía térmica, en función del costo del gas natural y del glicerol metilado (glicerol bruto) se muestran en la Fig.2.

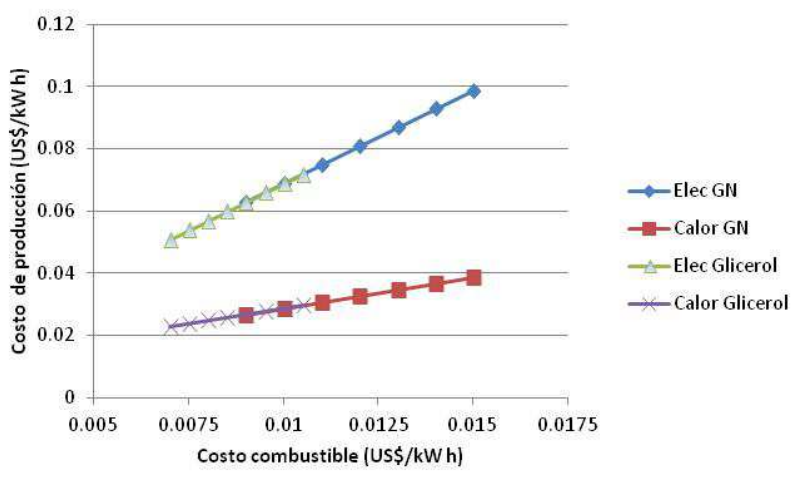

Figura 2: Costos de producción de electricidad y calor utilizando gas natural y glicerol bruto.

El precio del glicerol es una variable que tiene un gran impacto en la producción combinada de electricidad y calor. Dado que el glicerol, en la actualidad, tiene un precio bajo comparado al gas natural, proporciona menores costos de producción. Pero el uso del glicerol como combustible es dificultoso, porque aparecen problemas en la combustión, debido a la viscosidad que dificulta la atomización y posee un alto punto de ignición, que genera una llama fría con contenido de sales, que produce corrosión en los elementos de la caldera.

Además, como la producción total de electricidad es mayor que las demandas internas, existe un excedente de aproximadamente $13.75 \mathrm{MW}$. Este excedente de electricidad puede ser vendido a la red, ayudando a la economía de producción del sistema energético, haciéndolo económicamente más viable.

\section{Conclusiones}

Para cualquier proceso industrial, la integración de las relaciones cuantitativas que caracterizan el balance económico y el comportamiento energético de un sistema es una herramienta que permite la caracterización de los costos de los productos obtenidos en el proceso de producción basados en una propiedad medible como el contenido de energía, que es una magnitud termodinámica que obedece a leyes naturales.

Además de asegurar el abastecimiento de la electricidad y el calor para necesidades internas de la planta, existe un excedente de electricidad generada que puede ser vendido a la red, haciendo más viable económicamente el sistema energético propuesto.

En el presente caso, son comparados los costos de producción utilizando dos tipos de combustible: gas natural y glicerol.

Se debe destacar que los costos de generación de electricidad y de energía térmica (calor) son particularmente sensibless al precio de los combustibles, presentando las condiciones viables para utilizar el glicerol como combustible.

\section{Referencias}

[1] R. B. Evans; Thermoeconomic isolation and essergy analysis, Energy 5, 805 (1980).

[2] Y. M. El-Sayed y R. B. Evans; Thermoeconomics of heat power systems, J. Engineer. Power. 92, 27 (1970).

[3] Y. M. El-Sayed y R. A. Gaggiolli; A critical review of second law costing Methods I: Background and algebraic procedures, Transactions of the ASME, J. Energy Resources Technol. 111, 1 (1989).

[4] D. A. Sama; The use of second law of thermodynamics in process designs, Transactions of the ASME, J. Energy Resources Technol. 117, 179 (1995).

[5] G. Tsatsaronis; Thermoeconomic analysis and optimization of energy systems, Progress in Energy and Combustion Science, 19, 227 (1993).

[6] A. Bejan, G. Tsatsaronis y M. Moran; Thermal design and optimization, Ed. John Wiley \& Sons Inc., New York (1996).

[7] C. A. Frangopoulos; Optimal synthesis and operation of termal systems by the thermoeconomic functional approach, J. Engineer. Gas Turbines and Power, 114, 707 (1992).

[8] A. Lazzaretto y A. Macor; Direct calculation of average and marginal cost from the productive structure of an energy system, J. Energy Resources Technol. 117, 171 (1995).

[9] A. Franco y C. Casarosa; Thermoeconomic evaluation of the feasibility of highly efficient combined cycle power plants, Energy 29, 1963 (1987).

[10] J. L. Silveira; Cogeração disseminada para pequenos usuários: estudo de casos para o setor terciário, Tese de doutorado, Universidade Estadual de Campinas, UNICAMP, Campinas (1994). 
[11] C. E. Tuna; Um método de análise exergoeconómica para otimização de sistemas energéticos, Tese de doutorado, Faculdade de Engenharia de Guaratinguetá, FEG, Guaratinguetá (1997).

[12] J. A. P. Balestieri; Cogeração: geração combinada de eletricidade e calor, $1^{\mathrm{a}}$ edição, $279 \mathrm{p}$, Ed. da UFSC, Universidade Federal de Santa Catarina, Florianópolis (2002).

[13] A. Demirbas; Importance of biodiesel as transportation fuel, Energy Policy 35, 4661 (2007).

[14] N. Rahmat, H. Z, Abdullah y A. R. Mohamed; Recent progress on innovative and potential technologies for glycerol transformation into fuel additives: A critical review, Renewable and Sustainable Energy Reviews N 14, 987 (2010).

[15] J. C. Thompson, B. B. He; Characterization of crude glicerol from biodiesel production from multiple feedstocks, Applied Engineering in Agriculture $\mathbf{2 2}(2), 261$ (2006).

[16] M. Stelmachowski; Utilization of glycerol, a byproduct of the transesterification process of vegetable oils: A review, Ecological Chemistry and Engineerings 18(1), 9 (2011).
[17] M. Dasari; Crude glycerol potential described, Feedstuffs pp. 16, 16 de octubre (2007). Disponible en: http://fdsmagissues.feedstuffs.com/fds

/PastIssues/FDS7943/fds16_7943.pdf

[18] www.steamtablesonline.com, Steam Tables Online.

[19] G. J. Van Wylen, R. E. Sonntag y C. Borgnakke; Fundamental of Classic Thermodynamics, Book and Disk, 864 p., John Wiley \& Sons Inc., New York (2005).

[20] M. D. Bohon, B. A. Metzger, W. P. Linak, C. J. King y W. L. Roberts; Glycerol combustion and emissions, Proceedings of the Combustion Institute 33, 2717 (2011).

[21] R. F. Boehm; Design Analysis of Thermal System, Ed. John Wiley \& Sons Inc., New York (1987).

[22] J. L. Silveira; Estudo de sistema de cogeração aplicado a fabricação de papel e celulose, Dissertação de Mestrado, Escola Federal de Engenharia de Itajubá, EFEI, Itajubá, MG (1990).

[23] D. W. Garagatti Arriola; Sistema de tri e tetra combinado de cogeração: avaliação exergética e termoeconômica, Dissertación de Maestría, USP, 206 p., São Paulo (2000). 